\title{
Wall and Plasma Membrane Structures of Hydrogenomonas eutropha as Revealed by Stereography of Replicas from Complementary Freeze-etched Surfaces
}

\author{
BY B. J. VELTRI AND J. H. MCALEAR \\ Department of Biology, \\ The Catholic University of America, \\ Washington, D.C. 20017, U.S.A.
}

(Accepted for publication 24 September 197I)

SUMMAR Y

\begin{abstract}
A method is described for fracturing frozen samples of Hydrogenomonas eutropha (HI6 PHBA-4) so that both fracture faces can be studied by making platinum shadowed carbon replicas and locating complementary areas in each, employing the transmission electron microscope. Complementary areas studied by stereo microscopy showed complementary height differences in the corresponding replicas. Three pairs of complementary fracture faces had projections on the outermost faces of the inner layers of the plasma membranes which pulled out from the adjacent layer. Patches, bare of projections, on this surface had a complementary material of unknown function opposite.
\end{abstract}

\section{INTRODUCTION}

Hydrogenomonas eutropha is a Gram-negative facultative autotrophic bacterium which utilizes molecular hydrogen as its energy source when growing autotrophically. It can also oxidize molecular hydrogen concurrently with an organic substrate for energy. Hydrogenomonas eutropha (HI6 - wild-type) normally synthesizes abundant amounts of poly-betahydroxybutyric acid (PHBA). A mutant strain (HI6 PHBA-4) isolated by Schlegel, Lafferty \& Krauss (1970) which does not produce PHBA was used in the present study.

Freeze-etching of biological samples was introduced by Steere (1957), improved by Moor, Muhlethaler, Waldner \& Freys-Wysslling (196I), and simplified by Bullivant \& Ames (1966). McAlear \& Kreutziger (1967) also employed freeze-etching in their studies and redesigned the Bullivant \& Ames device to permit freeze-etching by the use of radiant heat applied to the fractured surface of the frozen sample (Kreutziger \& McAlear, 1967; McAlear, I968).

The uses of stereo electron microscopy have been reviewed by Gray \& Willis (I968) and stereoscopy for observing freeze-etch replicas has been described by McAlear, Shatos, Dempsey \& Veltri (1969). Advances in the technique of producing freeze-etched replicas, employing a double shroud mechanism, have been made by Steere (1969). Chalcroft \& Bullivant (1970) modified their freeze-fracture technique to permit observation of complementary replicas.

The purpose of this study was to demonstrate both sides of fractured surfaces of the wall and plasma membrane of Hydrogenomonas eutropha (HI6 PHBA-4), by employing simple techniques with a modified Berkeley freeze-etch cold block device. Some of the fractured surfaces are illustrated in stereo by taking micrographs at two different angles for each complementary replica so that complements have a similar stereo direction and degree of stereo separation. 
Ai Surface of ice.

ABBREVIATIONS

Ao Outer surface of the organism.

$\mathrm{Bi}$ Inner facing surface of the thin outer component.

Bo Theoretical complement of the Bi surface - not yet identified.

$\mathrm{Ci}$ Inner facing surface of the second layer of the cell wall.

Highly ordered array - with about $7 \mathrm{~nm}$ unit periodicity.

Co Complement to $\mathrm{Ci}$ - outer facing surface of the third layer of the cell wall.

Di Inner facing surface of the innermost layer of the cell wall. Seen only in small steps.

Do Theoretical complement to the Di surface - not yet characterized.

Ei Inner facing surface of the outer half within the plasma membrane, characterized by irregular pits and granules.

Eo Outer surface of the inner component of the plasma membrane. Granular appearing surface sometimes closely packed on a substrate which has approximately $5 \mathrm{~nm}$ unit periodicity in two directions, nearly at approximately right angles.

Fi Inner facing surface of the plasma membrane. Thus far, noted only as regions of oblique fracture through the cytoplasm, or areas lying upon the Ei surface, but larger than patches from within the membrane.

Fo Etched surface of the cytoplasm. No regular structure. Highly irregular granules.

$\mathrm{Pa}$ Patches - found within the plasma membrane lying on the Ei surface. Showing approximately 5 to $7 \mathrm{~nm}$ unit spacing.

Pe Pegs - found at fractured junction of Ei and Eo. Originating across the innermost component of the plasma membrane and pulling out of the Ei surface.

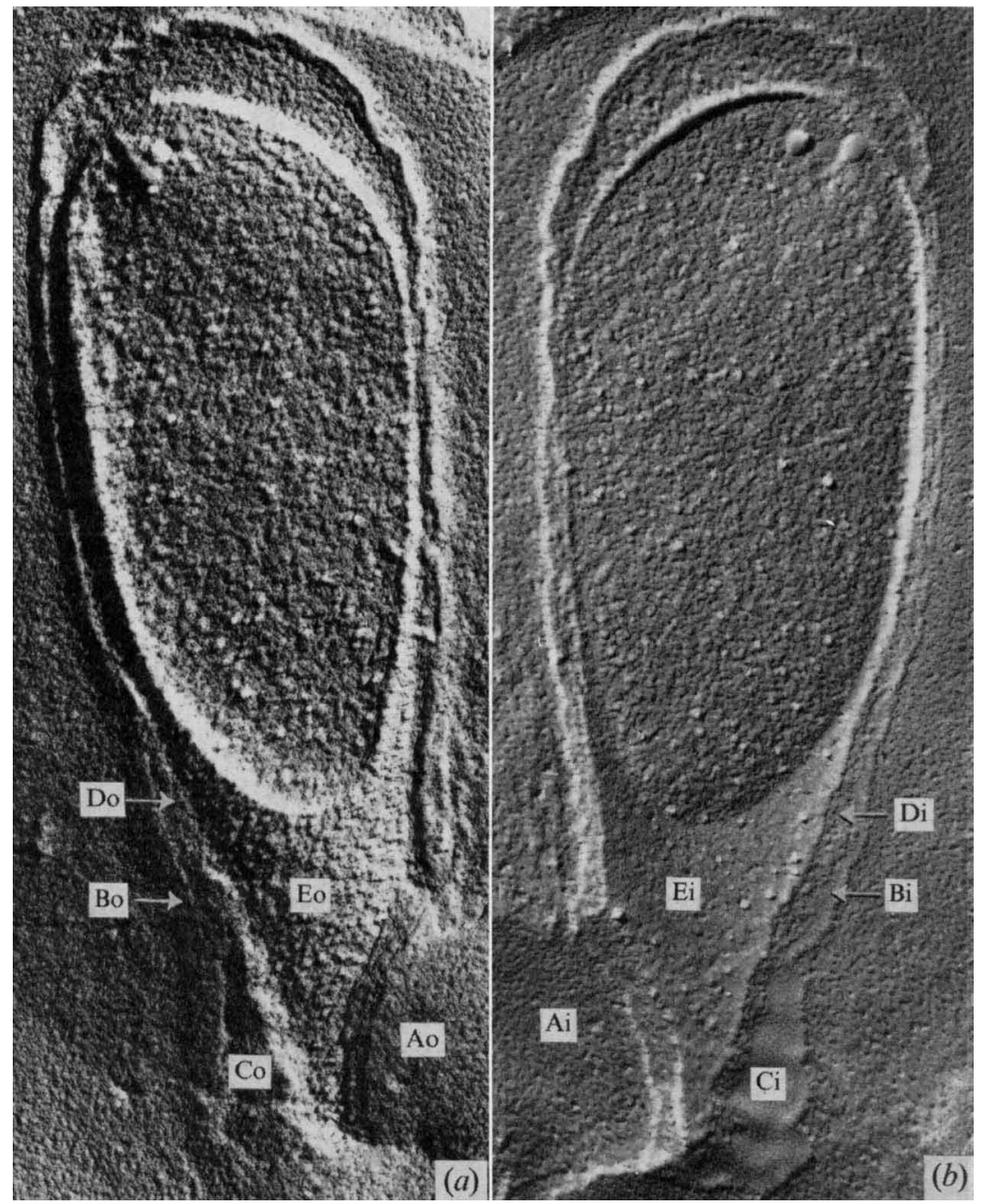

Fig. I (a) Demonstrates Ao, Bo, Co, Do and Eo surfaces. $\times$ 100,000. $(b)$ Demonstrates complementary surfaces $\mathrm{Ai}, \mathrm{Ci}$ and $\mathrm{Ei}$. The $\mathrm{Bi}$ surface appears as a narrow ridge and the $\mathrm{Di}$ surface is noted as an edge. $\times 100,000$. 

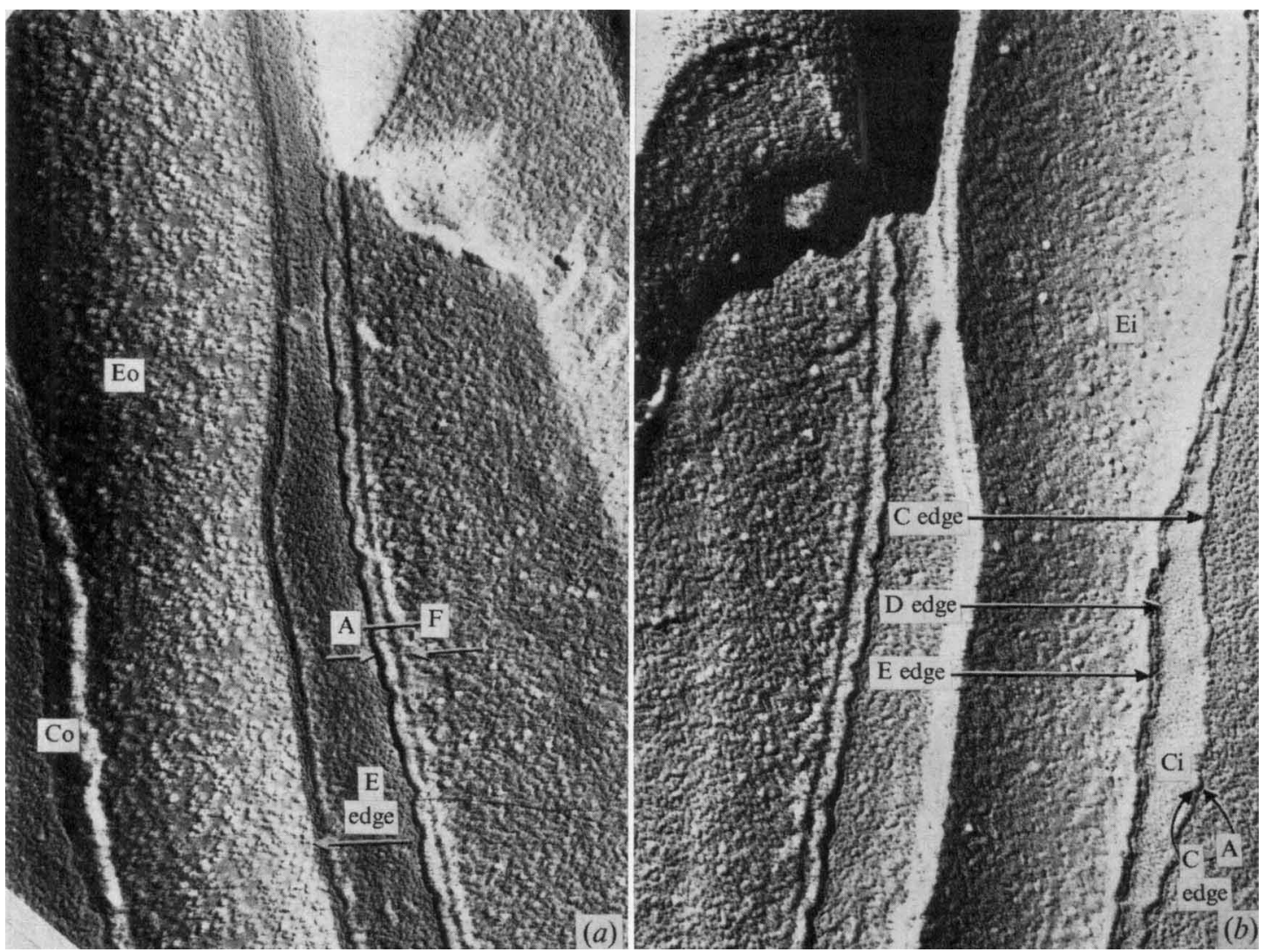

Fig. 2 ( $a$ ) Demonstrates A-F steps, E edge, Co and Eo surfaces, $\times 100,000$. (b) Complement to $2 a$ revealing $\mathrm{A}, \mathrm{C}, \mathrm{D}$ and $\mathrm{E}$ edges. The $\mathrm{Ci}$ and $\mathrm{Ei}$ spacing shows a step which must be the $\mathrm{D}$ fracture plane - giving a glimpse of the Di surface at this edge. $\times 100,000$.

\section{METHODS}

Hydrogenomonas eutropha (HI6 PHBA-4) was grown in tryptic soy broth (TSB - Difco) overnight at $30^{\circ}$ in an incubator shaker, then washed several times in $0.04 \mathrm{M}$-phosphate buffer $(\mathrm{pH} 7 \cdot \mathrm{I})$ containing $28 \%(\mathrm{v} / \mathrm{v})$ glycerol. Approximately three to four drops of concentrated erythrocytes (turkey, goose or chick cells) were mixed with the packed bacteria to serve as markers to locate adjacent bacteria in complementary replicas (R. L. Steere, personal communication).

Grid tubes ( $\mathrm{mm} \times 3 \mathrm{~mm}$ ) were made from copper grids ( 300 mesh, $3 \mathrm{~mm}$ diam.) by rolling them with an angled dissecting needle. A median horizontal cut was made on the tube so that it acted as a guide to facilitate cleaving when frozen. A minute area was not cut and acted as a hinge so that both halves could be folded back after freeze-cleaving.

Tubes were loaded with the mixture of bacteria and red cells, immersed in liquid freon for 5 to Io $s$ and then plunged into liquid nitrogen. The frozen specimen was then cleaved at the precut midline, folded back side by side and transferred to a specimen holder located in a modified Berkeley freeze-etch device. This entire device was then taken out of the nitrogen and quickly placed in an NRC vacuum evaporator and the pressure was reduced to $2 \times 10^{-8}$ Torr. Temperature was increased from $-190^{\circ}$ to $-100^{\circ}$ and held there for 2 min to permit 
etching of the specimen. The cleaved-etched sample? was then shadowed with a carbonplatinum pellet and carbon coated for backing.

The fractured replicated specimen was then removed from the degassed evaporator and placed in separate wells of a porcelain spot plate. Replicas were cleaned with dilute to concentrated 'Clorox' (sodium hypochloride solution), rinsed several times with distilled water and mounted on one copper grid (100 mesh, 2.3 mm) supported by formvar. Replicas were examined with an AEI 8or electron microscope equipped with a $\pm 30^{\circ}$ double tilt stage at an initial magnification of 40,000 . Replicas were tilted $20^{\circ}$ between micrographs using combinations of the $\mathrm{X}$ and/or $\mathrm{Y}$ drives to approximate similar separation and direction in each complement. Further details of our methods are given by Veltri \& McAlear (I97I).

Micrographs in Fig. I, 2, 6 and 7 were mounted as complementary pairs. Micrographs in Fig. 5 were mounted so that they appear as two stereo pairs horizontally and complements vertically. The stereo separation must present the surfaces with the correct sign and the shadowing angle ought to verify this. The stereo angle which is optimum for a given magnification and thickness has been described by Hudson \& Makin (1970).

The diagram for Fig. 5 demonstrates the surfaces since markings on the prints would interfere with the stereo effect. A simplified technique employing a glass slide, cover slip and immersion oil has been devised by Gray \& Willis (1968) for observing stereo pairs.

\section{RESULTS AND DISCUSSION}

A lettering code has been devised to describe various fractures, surfaces and layers seen in the diagrams and plates. The fracture plane is the zone of least resistance to cleavage. Fractures A, B, C, D, E and F are illustrated in Fig. I and 4. The A fracture plane reveals the Ai surface (ice) on one replica and the Ao (outer surface of the cell wall) on the complementary replica. Similarly the Bi surface reveals the inner face of the outer component of the cell wall on one replica and the Bo surface reveals the outer face of the second component of the cell wall on its complementary replica. Other fracture planes can easily be noted from Fig. I. Figure 2 demonstrates the surfaces as observed from the micrographs. From complementary replicas the surfaces illustrated in Fig. I can be identified. In Fig. I $a$ there are a number of steps between the Ao surface and the next cell in the same micrograph. The complement (Fig. I $b$ ) shows corresponding steps between the Ei surface and the surface of the ice, Ai. Further, the Ai surface resembles that of the adjacent ice. Its intersection with its adjacent cleaved ice does not show a membrane-like edge, but such an edge is visible between the Ao face and its adjacent cleaved ice.

There are three major fracture planes - $\mathrm{Ao}$ and $\mathrm{Ai}$; $\mathrm{Co}$ and $\mathrm{Ci}$; $\mathrm{Eo}$ and $\mathrm{Ei}$ - and these are probably the zones of least resistance to cleavage. The Bi surface can be seen, as can the Fo and Fi surfaces and portions of the Di fracture. Edges cannot be observed clearly without stereo. The Bi edge in Fig. I $b$ cannot be seen without the use of stereo because it lies close to the limit of resolution of the technique.

The Ao-Bi layer is $\mathrm{I} \cdot 5$ to $2 \mathrm{~nm}$ in thickness (Fig. I). The Bo-Ci layer has a surface with an apparent hexagonal array of $7 \mathrm{~nm}$ periodicity. It is 3 to $3.5 \mathrm{~nm}$ thick (Fig. I, 2). The Co surface is the outer surface of the Co-Di layer of the wall which is the innermost layer(Fig. 2). The Co-Ci fracture plane may be close to the centre of the wall. The pattern of organization on the $\mathrm{Ci}$ surface is not matched on the complementary Co face which is seen clearly in, Fig. I $b$ and $6 a$; there is no clear evidence of a complementary ordered array as noted in the Ci face (Fig. I $a, 6 b$ ). The Co surface is easily distinguished from the Eo surface because of the 
Ultrathin section model of cell envelope of $H$. eutropha

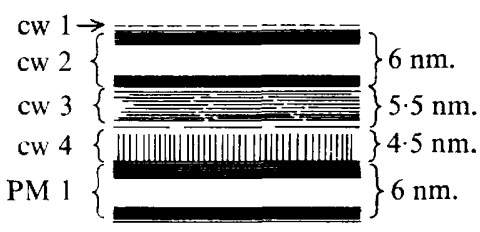

cw 1 Loosely bound layer cw 2 Triple layered membrane cw 3 Homogeneous layer cw 4 Peptidoglycan region cw 1-4 Cell wall complex

PM 1 Plasma membrane (triple layered)
Freeze-cleaved and etched model of cell envelope of $H$. eutropha

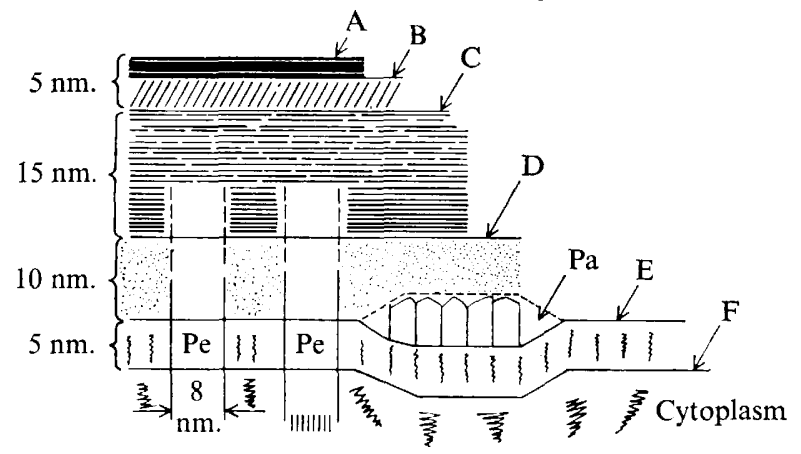

A-B Outer component of cell wall

B-C Second component of cell wall

C-D Inner component of cell wall

A-D Cell wall complex

D-E Outermost component of plasma membrane

$\mathrm{E}-\mathrm{F}$ Innermost fracture of plasma membrane

D-F Plasma membrane

Pe Pegs

$\mathrm{Pa} \quad$ Patches

Fig 3.

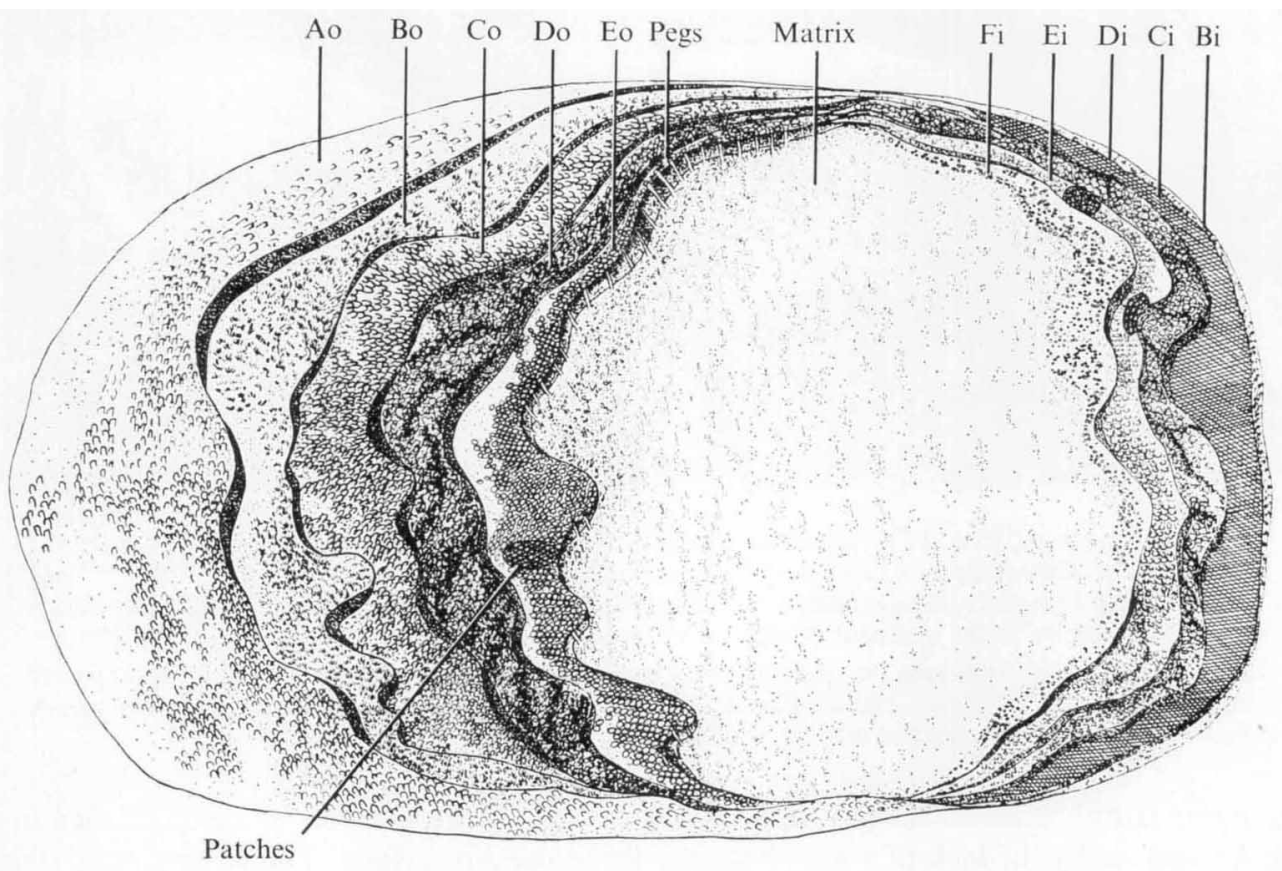

Fig. 4. Model of fractured specimen revealing surfaces, layers, patches and pegs as described in Abbreviations. 


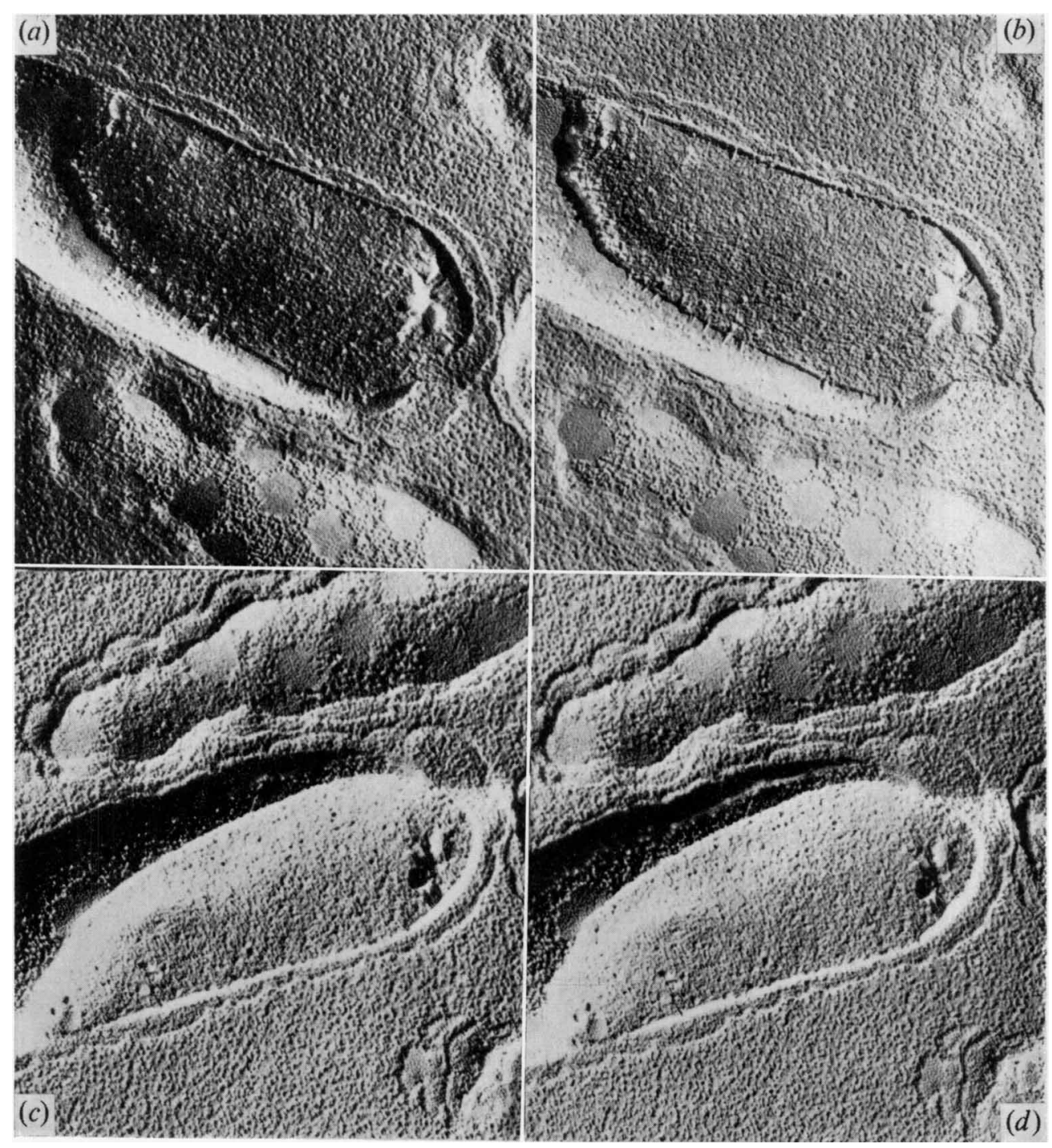

Fig. $5(a)$ and $5(b)$. Ei and Eo faces respectively demonstrate complementary 'patches'. On the Eo surface these regions appear devoid of bumps, but with a repeating surface pattern of 5 to $7 \mathrm{~nm}$ units. On the Ei surface complements to these areas show a material lying above the substrate which is complementary to the adjacent bumps. $\times 75,000$.

Fig. $5(c)$ and $5(d)$. Indicates the origin of these bumps where pegs across the Fi and Eo layer (inner component of the PM) and into the Ei face can be noted at the junction of the $\mathrm{E}$ fracture plane. A mesosome is noted within the cell. $\times 75,000$.

small step from the ice to the Co surface. The Co surface can likewise be distinguished from the Ao surface by the lack of a step from the ice to the Ao surface. This is confirmed where there is no step from the ice surrounding the organism to the Ai surface (Fig. I $b$ ). The Ai surface must therefore be the surface of the ice adjacent to the cell wall. The inner facing surface can be similarly distinguished since there is a relatively small step from the ice to the Ci surface and a large step from the ice to the Ei surface. The Co surface then would seem to be the outer surface without a distinctive structure, in contrast to the $\mathrm{Bo}-\mathrm{Ci}$ layer. In 


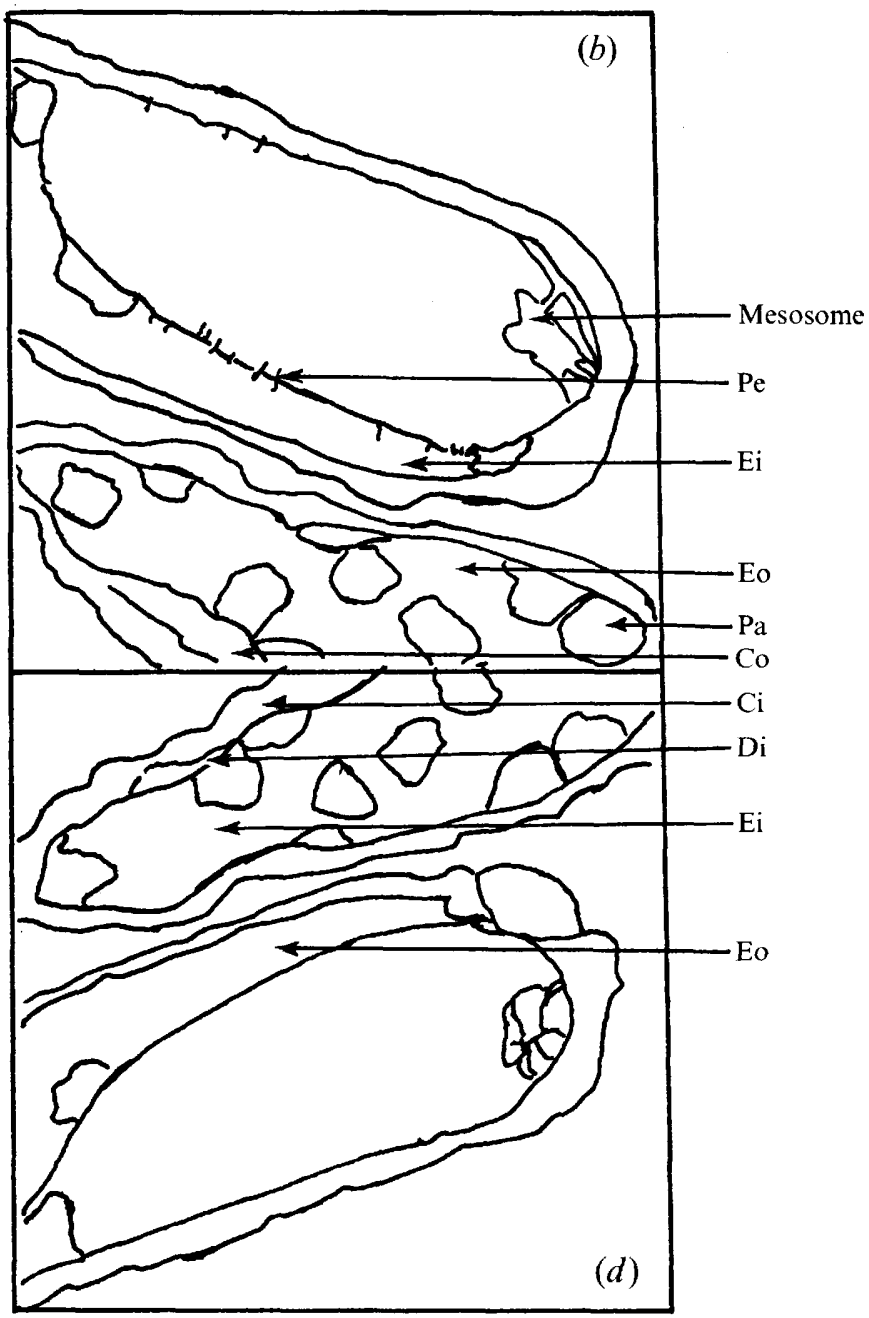

Fig. 5. Diagram.

Fig. I $b$, the inner facing fracture shows a series of irregular steps across this component going from the $\mathrm{Ci}$ surface up to the Ei surface. In Fig. $6 a$ and $6 b$, there is a region where there is an in-fracture through the Co layer and the corresponding complementary surface, which pulls out of this in-fracture. This is the up-fracture for that in-fracture. This could approach the surface of the plasma membrane, but if it does so, it would suggest that the face of the plasma membrane does not show any regular pattern of organization. The impression of the fracture across this third layer of the complex is that of an irregular longitudinal stratified shale. The corresponding outer facing surfaces related to the Di (i.e. the Do surface) may be seen in a small area on the outer facing component in Fig. $\mathrm{x} b$, but demonstrates no distinct structural pattern.

The most common fracture plane is the $\mathrm{E}$ which demonstrates two dissimilar surfaces, the Ei and Eo. The inner facing fracture surface (Ei) is an irregularly mottled array of bumps and pits. The fairly uniform bumps on the Eo surface do not closely correspond to the pits in the Ei surface. This may be explained by the shadowing artifact which emphasizes bumps 


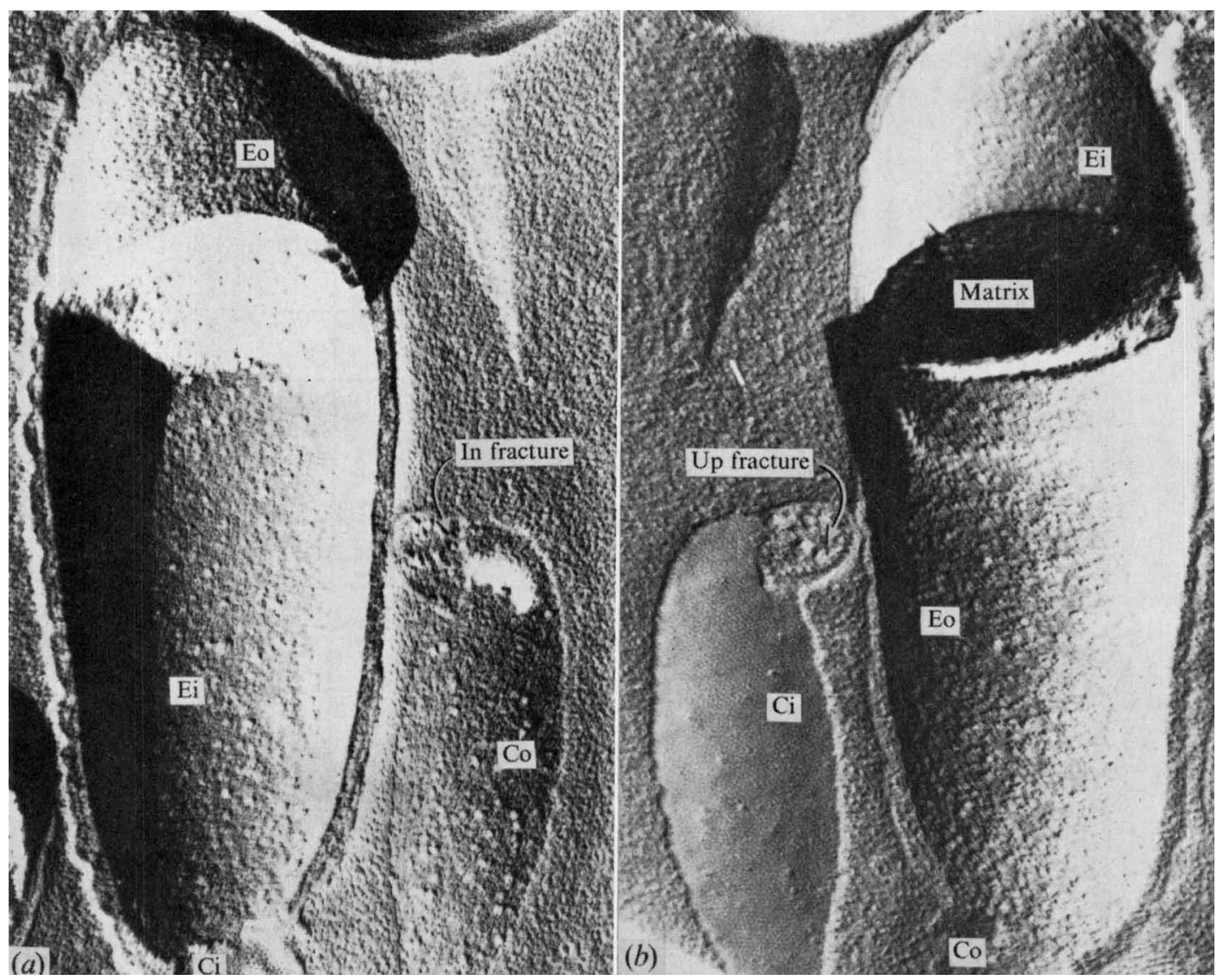

Fig. 6 (a) demonstrates different fracture planes in two micro-organisms. An 'in-fracture' and Co surface are revealed in one micro-organism. Larger micro-organism reveals the $\mathrm{Eo}, \mathrm{Ei}$ and $\mathrm{Ci}$ surfaces. $\times$ I00,000.

Fig. $6(b)$ Complement to $4 a$ revealing an elevated region on the $\mathrm{Ci}$ face. Note complementary indentations in the Co face in $4 a$. These may reflect the rather structureless Co-Di layer or may approach the Do face (outer surface of PM). $\times 100,000$.

and minimizes pits. These bumps may be the ends of pegs, since they occur at the junction of the fracture across the cytoplasm and the Eo to Fi layer and along the Eo surface. The pegs appear to arise from the cytoplasm through the Eo-Fi layer (Fig. 3, 4). Since the distribution of the pegs are observed on edge (Fig. $5 a, b$ ), one would expect these pegs to appear as bumps on the Eo surface. The pegs on this surface appear to be in the process of being pulled out by the fracture of the Ei surface.

Pegs seem to come through the inner half of the plasma membrane. It would seem that the pegs do indeed pull out of the Do-Ei layer during fracture (Fig. $5 a, b$ ). They are about $8 \mathrm{~nm}$ in diameter, and may be more than $15 \mathrm{~nm}$ long, indicating that their total length may be considerably greater than the thickness of the plasma membrane. Although some of this apparent length could be due to a distortion at the cleavage plane (i.e. a stretching), it is a little difficult to imagine this occurring at the temperature in which the fractures occurred and were replicated. The structures should be reasonably rigid at $-190^{\circ}$ to $-100^{\circ}$. These 


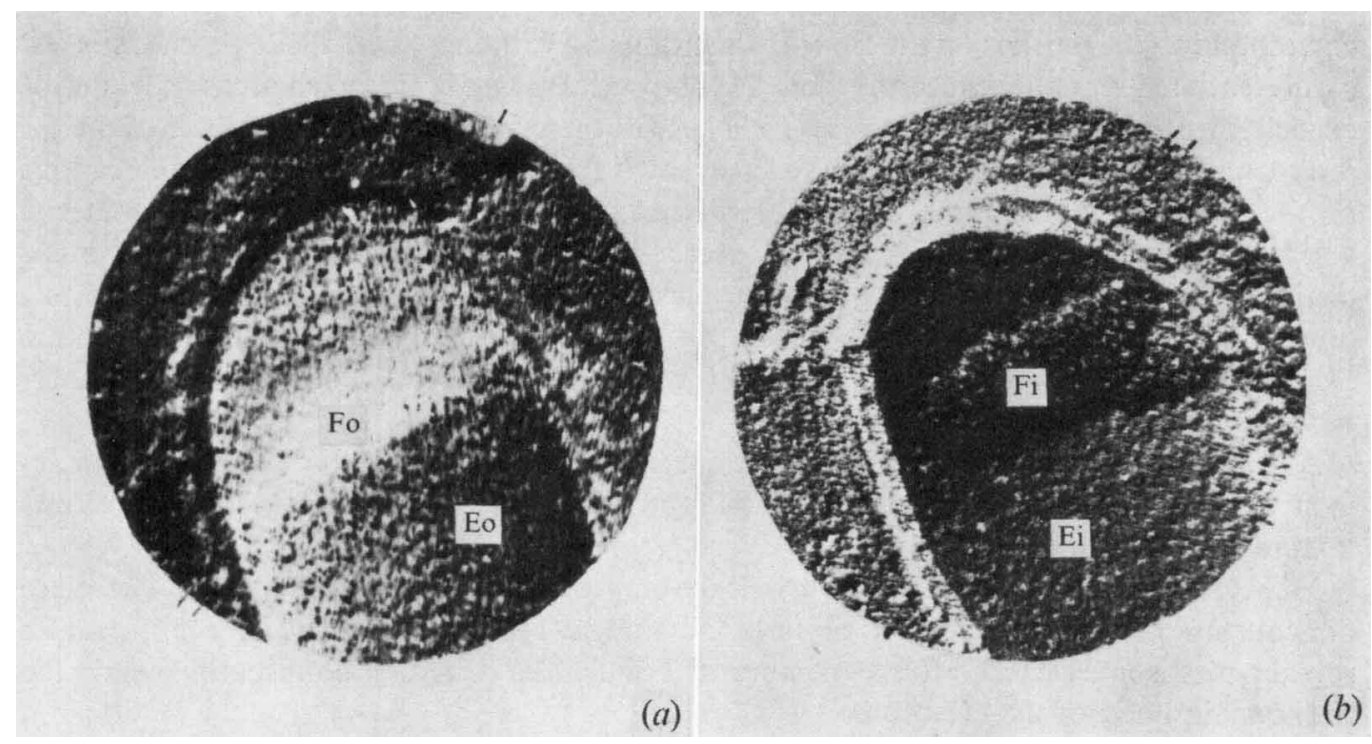

Fig. 7 ( $a$ ) demonstrates an etchable surface, Fo - as one would expect if the cytoplasmic matrix were thus laid bare. The Eo surface is also shown. $\times 100,000$.

Fig. $7(b)$ The Fi face is indicated in this complement by a layer lying upon the Ei surface. There is no regular structure apparent. $\times 100,000$.

pegs do appear real and are consistently found in many different organisms (Fig. $5 a, b$ ). It is probable that these are, in fact, transmembrane elements. Burdett \& Rogers (unpublished data) have found pegs in sections connecting the outer surface of the membrane with the cell wall. Areas of adhesions have been demonstrated between retracted cytoplasm and cell walls of Gram-negative bacteria (Bayer, 1968). There seems to be no doubt that the walls and membranes of bacteria are firmly pegged together. At the molecular level, this could be the result of the presence of enzymes polymerizing peptidoglycan in the membrane (Rogers, I970). The polymerized strands remain attached to the enzyme sites as they wind off into the wall.

The inner half of the plasma membrane, (the Eo-Fi layer) is observed principally as an outer facing surface (Eo). The inner face of this can be seen only in Fig. $2 b$ where a patch of material is observed as the Fi surface and where it follows the surface fairly regularly. This would be clearly demonstrated stereoscopically. It is visible as a rather thin layer corresponding to about the thickness expected from the inner half of the plasma membrane, where it shows no pattern of organization similar to that of the Ei surface. The Fi surface could be indistinct due to association with cytoplasmic material. This would be expected from Fig. $5 a$ and $b$, where the base of the pegs is observed apparently arising below the PM and penetrating through it. One might expect, therefore, more protein to be associated with the inner surface of the PM, perhaps not in a structurally organized manner that could be recognized with freeze-etching but, nevertheless, in such a way as to obscure otherwise detectable detail of the inner facing surface.

In some preparations (Fig. $5 a, b, c, d$ ), the Eo surface shows patches where there are no outer facing granules and in which the substrate appears, superficially, to be smooth. However, in more detail it shows an oriented parallel periodicity of about $7 \mathrm{~nm}$ centre to centre. The complementary Ei surface shows a similar periodicity, but usually at about $90^{\circ}$ 
to the direction of periodicity on the Eo surface. These patches on the Ei surface clearly correspond to the patches on the Eo surface fitting one with the other. These patches suggest a differentiated region because: (i) there are no peg scars on the Eo patch areas as would be expected from pegs traversing the Eo-Fi layer; (ii) there is no step from the level of the Eo interpatch or bump areas to the patch surface; and (iii) there are also corresponding elevations on the Ei surface patch areas. This suggests that patches represent an interstitial material and are distinct from the adjacent surface areas. We further suggest that the Eo-Fi layer is a distinct layer and not the surface of the cytoplasm because: (i) the Eo patch surface shows a fine regular structure unlike the cytoplasm; (ii) a distinct fracture edge occurs between the Eo surface and across the cytoplasm; and (iii) another surface also observed is the innermost, or on top of the Ei surface.

At present little can be inferred from the $\mathrm{F}$ fracture plane (Fo-Fi). However, the complement (Fo) to the presumed Fi surface does appear like the cytoplasm (Fig. 7a), both in its texture and its depth of etching.

Most Gram-negative bacteria have well-developed mesosomes (Ryter, 1968). These are difficult to detect in freeze-etched replicas (Nanninga, 1968). However, Fig. $3 a, b, c$ and $d$ reveal a mesosome attached to the membrane. Tubule-like structures connect the membrane to the main body of the mesosome.

\section{CONCLUSIONS}

The observations made by application of the freeze-etching technique confirm the concept of the multilayered structure of the Gram-negative cell envelope. Freeze-etching reveals new information and avoids shrinkage and other artifacts caused by other methods. Double replicas help interpretation by providing positive identification of complementary surfaces. In addition, stereoscopic analysis of replicas provides a better guide to relative elevation than shadowing, and provides more information, better resolution, and identification of replica artifacts. Artifacts produced by freeze-etching, however, are different from those of other methods.

\section{Cell wall and plasma membrane}

There is evidence of a very thin outer layer approximately $\mathrm{I} \cdot 5$ to $2 \mathrm{~nm}$ thick. Below that there is a layer which reveals a face with a highly ordered periodicity of about 3 to $3.5 \mathrm{~nm}$ and possible hexagonal array. This layer would seem to correspond to the lipopolysaccharidelipoprotein region described by other investigators. The next layer demonstrates little structural detail and probably corresponds to the peptidoglycan region. The portion affected by lysozymes and proteolytic enzymes does not appear in these studies as a distinct region, nor is the outer surface of the plasma membrane visible.

The surface revealed by the most common fracture plane (E) shows little correspondence to those of the membrane of higher cell types. In particular, the pegs extending from the cytoplasm and probably through the plasma membrane, are unique. The substrate for the pegs.noted on the Eo surface has a regular periodicity unlike that observed in higher celltype membranes, but perhaps more similar in dimension to the gas vacuole membrane of the blue green algae (Cohen-Bazire \& LeFor-Tran, 1968). The periodicity of the Eo-Fi layer seems to be about in the same order as the thickness of the non-unit membrane of the gas vacuoles of blue green algae. The minimum spacing between the pegs would also suggest a separation of the pegs from each other by same dimension, 7 to $8 \mathrm{~nm}$.

The patches which we have observed, as well as the pegs, must have some function. Pegs have been noted by other investigators who suggest that these serve to hold the wall and 
membrane components of bacteria together. But the function of the patches is unknown and these were not observed in all preparations.

The anatomical detail of the bacterial membranes described suggest a different basic structure from that of any higher cell type, or any of the eukaryotic cell types described thus far. Need for further work employing chemical and enzymatic degradation of the cell wall, co-ordinated with freeze-etching, is indicated. It may also be of value to compare organisms in different metabolic states. Suggested technical refinements could well include use of ionetching to reveal surfaces not yet exposed by fracture.

We are grateful to Dr H. G. Schlegel for the Hydrogenomonas eutropha mutant (HI6 PHBA-4), Dr D. C. Brungart for his advice, and the technical skills of Dr B. T. DeCicco. We are also grateful to Mrs B. Greene for the excellent diagrams, and to the staff of the medical audio visual services at the Walter Reed Institute of Research for the photography.

\section{REFERENCES}

BAYER, M. E. (1968). Absorption of bacteriophages to adhesions between wall and membrane of Escherichia coli. Virology 2, 346-356.

Bullivant, S. \& Ames, A. (1966). A simple freeze-fracture replication method for electron microscopy. Journal of Cell Biology 29, I76-I78.

Chalcroft, J. P. \& Bullivant, S. (1970). An interpretation of liver cell membrane and junction structure based on observation of freeze-fracture replicas of both sides of the fracture. Journal of Cell Biology 47(I), 49-60.

Cohen-Bazire, G. \& LeFor-Tran, M. (1968). Structure granulaire du chromatoplasma des cyanophycees. European Conference on Electron Microscopy, Rome II, 395-396.

Gray, E. G. \& Willis, R. A. (1968). Problems of electron stereoscopy of biological tissue. Journal of Cell Science 3, 309-329.

Hudson, B. \& MAKIn, M. J. (I970). The optimum tilt angle for electron stereomicroscopy. Journal of Physics E: Scientific Instruments 3, 3 I I.

Kreutziger, G. O. \& McAlear, J. H. (I967). Three dimensional images of cardiovascular elements with freeze-etching. Proceedings 25th Annual EMSA Meeting, pp. II8-I I9.

MCAlear, J. H. (1968). Progress in a simple cold block device for freeze-etching. Journal of Cell Biology 39, $88 a$.

McAlear, J. H. \& Kreutziger, G. O. (1967). Freeze-etching with radiant energy in a simple cold block device. Proceedings 25th Annual EMSA Meeting, pp. I $16-117$.

McAlear, J. H., Shatos, M., Dempsey, A. \& Veltri, B. J. (I969). Stereoscopic analysis of freeze-etch replicas. Proceedings of the Royal Microscopical Society 4, I6I-166.

Moor, H., Muhlethaler, K., Waldner, H \& Frey-Wysslling, A. (196I). A new freezing ultramicrotome. Journal of Biophysical and Biochemical Cytology ro, I-I3.

NANNINGA, N. (1968). Structural features of mesosomes (chondroids) of Bacillus subtilis after freeze-etching. Journal of Cell Biology 39, 25I-263.

Rogers, H. J. (1970). Bacterial growth and the cell envelope. Bacteriological Reviews 34, 194-2I4.

RYTER, A. (1968). Association of the nucleus and membrane of bacteria: a morphological study. Bacteriological Reviews 32, 39-54.

SChlegel, H. G., LAFFerTy, R. \& KRAUSS, I. (1970). The isolation of mutants not accumulating poly-betahydroxybutyric acid. Archiv für Mikrobiologie 7r, 283-294.

SteERE, R. L. (1957). Electron microscopy of structural detail in frozen biological specimens. Journal of Biophysical and Biochemical Cytology 3, 45-60.

SteERE, R. L. (1969). Freeze-etching and direct observation of freezing damage. Cryobiology 6, I37-I 50.

VelTRI, B. J. \& MCAlear, J. H. (I97I). A method for preparing complementary halves of freeze-etch replicas of micro-organisms for transmission electron microscopy. Journal of Microscopy 93, 191-196. 